\title{
Transport properties of chrysazine-type molecules
}

\author{
Angelica G. Zacarias · E. K. U. Gross
}

Received: 5 May 2009/Accepted: 2 November 2009/Published online: 22 November 2009

(c) The Author(s) 2009. This article is published with open access at Springerlink.com

\begin{abstract}
Electronic transport properties are presented for the three lowest-energy conformers of the chrysazine molecule. The current-voltage characteristics of these isomers are calculated for two different connections of the molecule to gold leads. The results for the various structures differ so significantly from each other that the specific conformer and its connection to the leads can be identified by its transport characteristics. The calculated transport properties suggest that chrysazine may be used as a molecular optical switch.
\end{abstract}

Keywords Quantum transport .

Density functional theory · Molecular electronics

Research in the field of molecular electronics has enjoyed increasing popularity in recent years. The ultimate goal, the design of electronic devices where the basic units, such as transistors, are single molecules, promises an enormous gain in miniaturization as well as clock speed. On the theoretical side, the generic task is to calculate the current-voltage

Dedicated to Professor Sandor Suhai on the occasion of his 65th birthday and published as part of the Suhai Festschrift Issue.

A. G. Zacarias - E. K. U. Gross

Institut für Theoretische Physik, Freie Universität Berlin,

Arnimallee 14, 14195 Berlin, Germany

A. G. Zacarias · E. K. U. Gross ( $)$

Max-Planck-Institut für Mikrostrukturphysik,

Weinberg 2, 06120 Halle (Saale), Germany

e-mail: hardy@mpi-halle.de; hardy@mpi-halle.mpg.de

URL: http://users.physik.fu-berlin.de/ ag-gross

A. G. Zacarias - E. K. U. Gross

European Theoretical Spectroscopy Facility,

http://www.etsf.eu/ characteristics of systems consisting of a single molecule sandwiched between two semi-infinite metallic leads. In the past decade, a combination of density functional theory (DFT) with Green's function techniques has been developed [1-10] by several groups and can now be considered the standard $\mathrm{ab}$ initio technique to tackle this task.

The basic idea of the method is as follows. Starting from the standard Kohn-Sham (KS) equation, each KS orbital is decomposed into a part that lives on the left lead (L), a part that lives on the right lead (R), and one that lives on the central region $(\mathrm{C})$. With the corresponding blocks of the KS Hamiltonian, the KS equation of the complete system reads as follows

$\left(\begin{array}{ccc}H_{\mathrm{LL}} & H_{\mathrm{LC}} & 0 \\ H_{\mathrm{CL}} & H_{\mathrm{CC}} & H_{\mathrm{CR}} \\ 0 & H_{\mathrm{RC}} & H_{\mathrm{RR}}\end{array}\right)\left(\begin{array}{c}\phi_{\mathrm{L}}^{(\mathrm{KS})} \\ \phi_{\mathrm{C}}^{(\mathrm{KS})} \\ \phi_{\mathrm{R}}^{(\mathrm{KS})}\end{array}\right)=\varepsilon\left(\begin{array}{c}\phi_{\mathrm{L}}^{(\mathrm{KS})} \\ \phi_{\mathrm{C}}^{(\mathrm{KS})} \\ \phi_{\mathrm{R}}^{(\mathrm{KS})}\end{array}\right)$

where the direct interaction between the right and left lead has been neglected. Introducing the KS Green's functions, $G_{\mathrm{L}}$ and $G_{\mathrm{R}}$, of the semi-infinite leads,

$\left(E-H_{\mathrm{LL}}\right) G_{\mathrm{L}}(E)=\mathbf{1}$

$\left(E-H_{\mathrm{RR}}\right) G_{\mathrm{R}}(E)=\mathbf{1}$,

the equation for the central part of each $\mathrm{KS}$ orbital can be written as follows:

$\left(H_{\mathrm{CC}}+H_{\mathrm{CL}} G_{\mathrm{L}}(E) H_{\mathrm{LC}}+H_{\mathrm{CR}} G_{\mathrm{R}}(E) H_{\mathrm{RC}}\right) \phi_{\mathrm{C}}^{(\mathrm{KS})}=E \phi_{\mathrm{C}}^{(\mathrm{KS})}$.

In this equation, the non-Hermitian operators

$\Sigma_{\mathrm{L}}=H_{\mathrm{CL}} G_{\mathrm{L}}(E) H_{\mathrm{LC}}$

$\Sigma_{\mathrm{R}}=H_{\mathrm{CR}} G_{\mathrm{R}}(E) H_{\mathrm{RC}}$

act as sources or drains for the electrons in the central part, thus representing the coupling of the central part to the 
leads. In the simplest approximation, $H_{\mathrm{LL}}$ and $H_{\mathrm{RR}}$ can be modeled by a diagonal matrix of the bulk metallic leads proportional to its local density of states. Once $\Sigma_{\mathrm{L}}$ and $\Sigma_{\mathrm{R}}$ have been calculated, the transmission function is obtained from

$T(E)=\operatorname{trace}\left(\Gamma_{\mathrm{L}} G(E) \Gamma_{\mathrm{R}} G(E)^{\dagger}\right)$,

where $G(E)$ is the KS Green's function of the complete system, and $\Gamma_{\mathrm{L}}$ and $\Gamma_{\mathrm{R}}$ are the non-Hermitian parts of the above self-energies:

$\Gamma_{\mathrm{L}}=-i\left(\Sigma_{\mathrm{L}}-\Sigma_{\mathrm{L}}^{\dagger}\right)$

$\Gamma_{\mathrm{R}}=-i\left(\Sigma_{\mathrm{R}}-\Sigma_{\mathrm{R}}^{\dagger}\right)$.

Once the transmission function is known, the currentvoltage characteristics follow from the Landauer formula

$I(V)=(2 e / h) \int \mathrm{d} E T(E, V)\left[f\left(E, \mu_{+}\right)-f\left(E, \mu_{-}\right)\right]$

where $f$ is the Fermi-Dirac distribution referring to the chemical potentials, $\mu_{+}$and $\mu_{-}$, given by the Fermi energy \pm half the applied voltage $\mathrm{V}$.

Upon applying a bias to the metal-molecule-metal system, the electrons go from one metallic lead to the other via the molecular orbitals. The latter are modified by the presence of the metallic contacts, an effect that has been studied both experimentally [11] and theoretically [12]. A conducting level corresponds to a molecular orbital that is delocalized along the metal-molecule-metal system, while a non-conducting level is a localized orbital, which cannot connect both ends of the molecule attached to the metallic tips. The energy of a molecular orbital with respect to the Fermi level of the metallic leads determines at which bias this orbital begins to contribute to the current. To assess in which way a given molecule can be used in a molecularelectronic device, it is therefore essential to first understand the electronic structure of the molecule in complete detail.

Since in some cases a minor structural change of the molecule may lead to a radical change in the shape of its molecular orbitals, the transport properties can as well be strongly affected by a small change in the geometry of the molecule. Consequently, it is very important that the electronic properties of the molecular devices are calculated not only for the fully relaxed structures, but also, during the relaxation process, some atoms of the metallic tips have to be included as part of the central region. This structure, consisting of the molecule plus a few metallic atoms at each side, will be called the extended molecule. The Hamiltonian matrix appearing in Eq. 1 for this extended molecule is obtained from standard quantum chemistry calculations. In our calculations, we use the Gaussian code [13] with the basis sets $6 / 31++\mathrm{G}^{* *}$ and LAN2DZ for the isolated and extended molecules, respectively. In the DFT calculations, we employ the B3PW91 [14-17] hybrid exchange-correlation functional.

In the following, we present calculations of the currentvoltage characteristics of chrysazine-type molecules $(1,8$ dihydroxyanthraquinone) using the formalism described above. The biological activity of this molecule is known [18] to be due to a proton transfer. In the present work, we investigate structures of the molecule with lower energy (than the proton-transferred structure), obtained by the rotation of the terminal hydrogen atoms of the molecule. These conformers, which have been found experimentally $[19,20]$, are expected to have rather different electronic states and hence we also expect rather different transport properties. We will first analyze in detail the electronic structure of the isolated and extended molecules and then present the transport properties.

Our calculations of the isolated chrysazine molecule reveal the existence of three stable conformers as shown in Fig. 1. Starting from the relaxed planar structure (a), we change the dihedral angle of one of the $\mathrm{H}$ atoms and, keeping the dihedral angle fixed, we relax all the other atoms and determine the ground-state energy. Then we rotate the $\mathrm{H}$ atom further and, keeping again the dihedral angle fixed, relax all the other atoms, etc. This produces the potential energy surface plotted in Fig. 2 from structure (a) to structure (b). Then, starting from structure (b), we change the dihedral angle of the second $\mathrm{H}$ atom, always determining the relaxed ground-state energy for fixed dihedral angle. This leads to the second part of the potential energy surface in Fig. 2, from structure (b) to structure (c). The barrier between the two stable structures (a) and (b) is $0.72 \mathrm{eV}$ while between the structures (b) and (c) it is $0.76 \mathrm{eV}$. Structures where a hydrogen is fully transferred to the quinone group are much higher in energy and do not show a local minimum in the potential energy surface.
Fig. 1 Stable chrysazine conformers found within density functional theory. Structure a has the lowest energy; the relative energy differences for $\mathbf{b}$ and $\mathbf{c}$ are 0.55 and $1.20 \mathrm{eV}$, respectively (a)<smiles>O=C1c2cccc(O)c2C(=O)c2c(O)cccc21</smiles>

(b)<smiles>O=C1c2cccc(O)c2C(=O)c2c(O)cccc21</smiles>

(c)<smiles>O=C1c2cccc(O)c2C(=O)c2c(O)cccc21</smiles> 


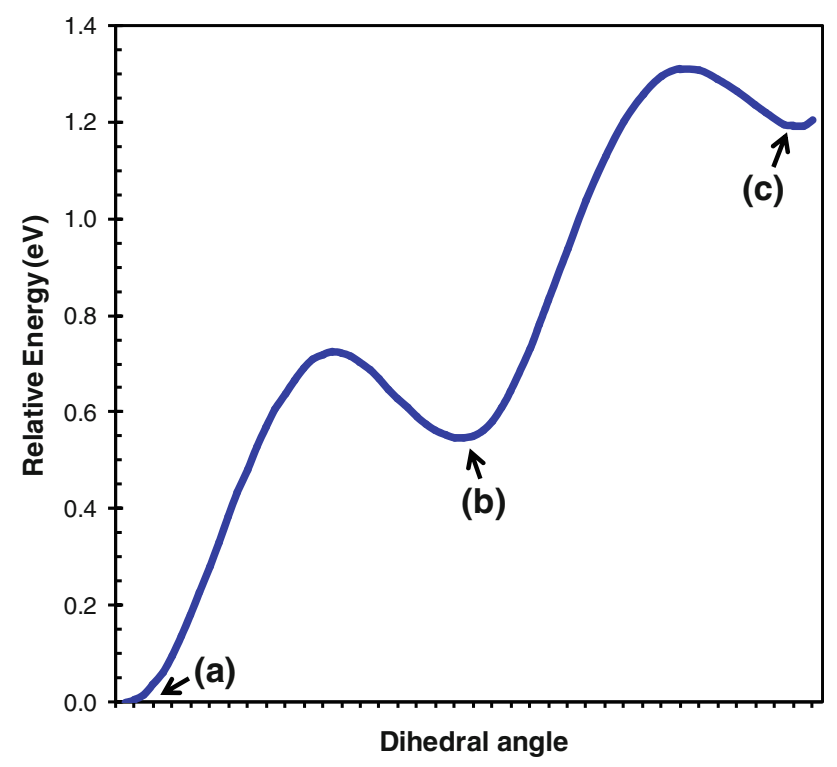

Fig. 2 Potential energy surface resulting from the free sequential rotation of the terminal hydrogen atoms of the chrysazine molecule. $a$, $b$ and $c$ correspond to the stable structures shown in Fig. 1

Figure 3 shows the molecular orbitals of the isolated chrysazine molecule. The highest occupied molecular orbitals (HOMO) of the three stable structures (a), (b) and (c) differ significantly from each other. In the case of structure (c), the HOMO is a $\sigma$ orbital, while for the structures (a) and (b) the HOMO is a $\pi$-type orbital. The charge distributions are drastically different in (a) and (b): The orbital is located at the two lateral rings in the case of the (a) conformer, while in (b) it is localized on that ring where the $\mathrm{H}$ has not been rotated with respect to structure (a). The lowest unoccupied molecular orbitals (LUMO) of these conformers, on the other hand, do not show strong differences.

To guarantee that no direct interaction between the terminal hydrogen atoms and the metallic tips takes place, we used linear spacers to link the chrysazine molecule to the metallic leads $(\mathrm{M}-\mathrm{S}-\mathrm{C} \equiv \mathrm{C}-$ chrysazine- $\mathrm{C} \equiv \mathrm{C}-\mathrm{S}-\mathrm{M}$ ). In this paper, we study two different ways to connect the molecule to the spacers (shown in Fig. 4). Clearly, one would expect that the conductance depends considerably on how the molecule is connected to the leads [21, 22]. On the left panels of this figure, the connection between the metallic tips and the chrysazine molecule is asymmetric and therefore there are two non-equivalent structures (b) and $\left(b^{\prime}\right)$ where only one hydrogen atom has been rotated. In the case of the symmetric connection (right-hand panels), there are only three stable conformers.

The DFT results show that for both types of connection, the lowest energy state corresponds to the conformer where both hydrogen atoms point to the central oxygen (isomers (a) in Fig. 4). The total energy of the asymmetric structure (a) is only $0.07 \mathrm{eV}$ higher than the energy of the symmetric structure (a). The asymmetric structures (b) and ( $\left.b^{\prime}\right)$ shown in Fig. 4 are 0.58 and $0.71 \mathrm{eV}$, while the asymmetric structure (c) is $1.25 \mathrm{eV}$ higher in energy with respect to the asymmetric structure (a). For the symmetric case, the relative energy of structure (b) is $0.72 \mathrm{eV}$, while (c) is $1.34 \mathrm{eV}$ higher in energy with respect to the symmetric structure (a).

Given the symmetry of the extended molecule, we observe that the location of the HOMO is regulated by the position of the hydrogen marked in Fig. 4 as $\mathrm{H}^{\prime}$. For the extended asymmetric structure, we find that not only the HOMO, but the LUMO orbitals as well are synchronized with the position of the atom $\mathrm{H}^{\prime}$ (see Fig. 5), that is, the rotation of $\mathrm{H}^{\prime}$ in structure (a) controls the redistribution of the HOMO and the LUMO from one side of the molecule to the opposite side (see HOMO and LUMO orbitals for (b) in Fig. 5). Likewise, when in the structure $\left(b^{\prime}\right)$ the second hydrogen atom is rotated toward the asymmetric structure (c), the HOMO and LUMO orbitals shift their densities to the opposite side of the molecule. The HOMO of the symmetric structure (a) is reasonably delocalized, while for
Fig. 3 HOMO (upper panel) and LUMO (lower panel) for the structures (a), (b) and (c). The HOMO of $\mathbf{a}$ and $\mathbf{b}$ is $\pi$ type, while the one of $\mathbf{c}$ is $\sigma$ type. The HOMO is delocalized in the outer rings for the structure (a), while for the structure (b) it is localized in one of the rings. The redistribution of this orbital is associated with the rotation of the terminal hydrogen atoms. The LUMO orbitals are largely independent of the rotation of the terminal hydrogen atoms (a)
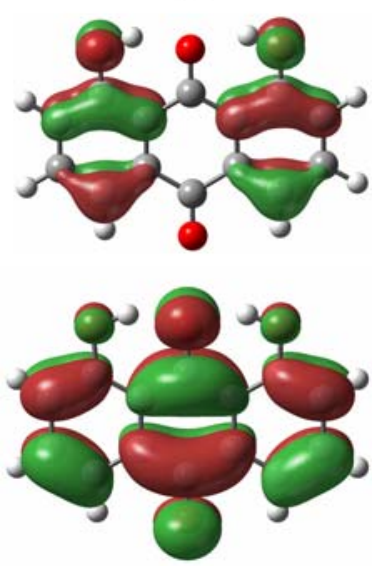

(b)
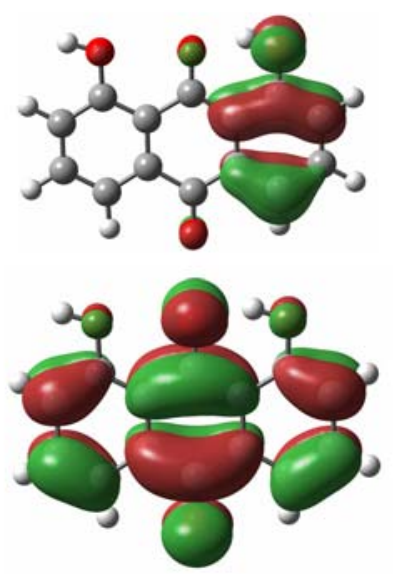

(c)

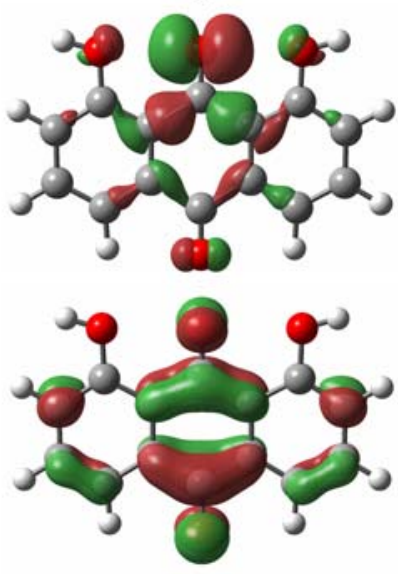


Fig. 4 Stable extended chrysazine conformers found within density functional theory. The atom marked as $\mathrm{H}^{\prime}$ represents the hydrogen that regulates the distribution of HOMO and LUMO (see Fig. 5)

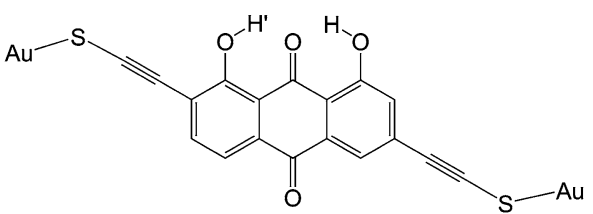

(a)
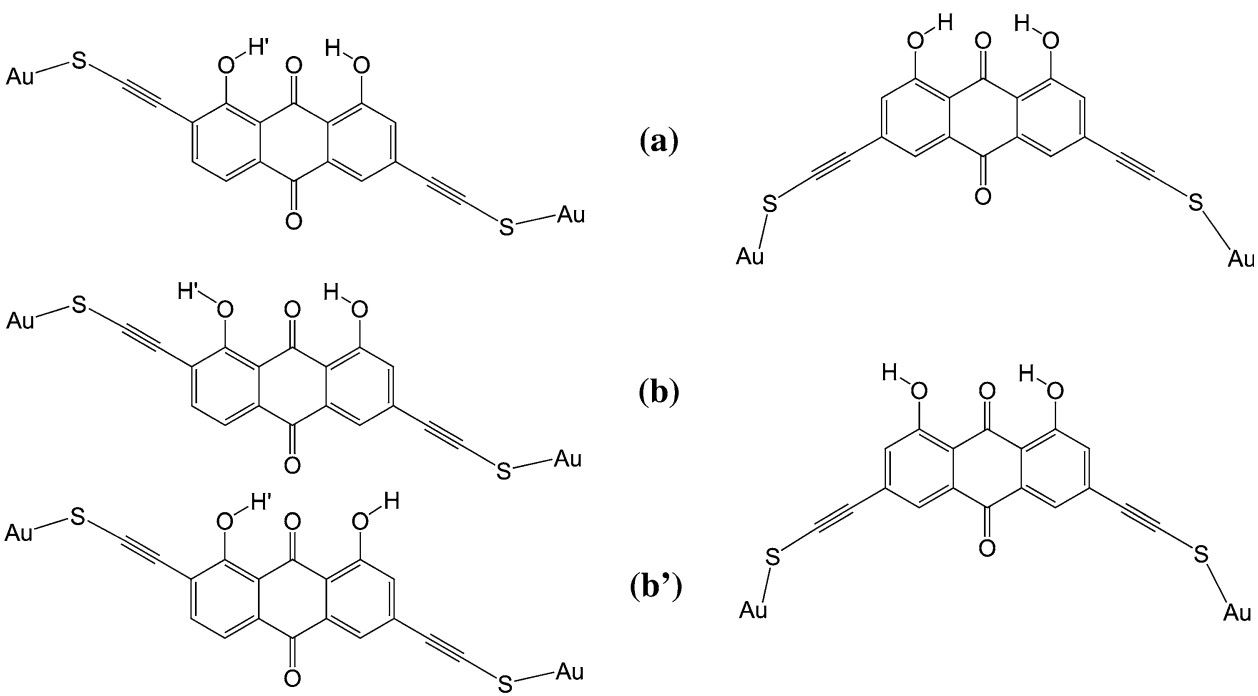

(b)

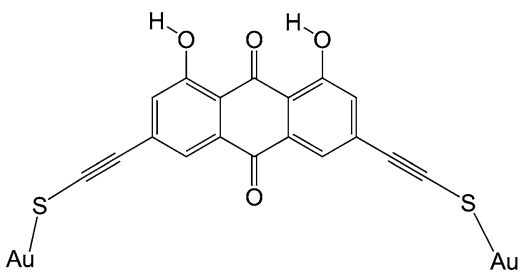

(c)

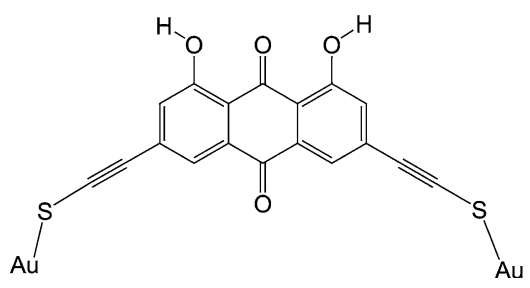

the symmetric structure (b), this orbital localizes, similar to the asymmetric structure (b), on one side of the molecule. The rotation of the second hydrogen atom, from structure (b) to structure (c), for these systems delocalizes the HOMO orbital along the whole molecule. None of the LUMO orbitals of the symmetric structures are affected by the rotation of the terminal hydrogen atoms.

The observed correlation between the rotation of the hydrogen atoms and the distribution of the HOMO and LUMO orbital density is a first indication that the transport properties for these systems will be significantly different. In Fig. 6, this correlation is shown for the conformer (a), both for the asymmetric and the symmetric connections. The energy axis is centered to the Fermi energy of bulk gold. The plots in this figure illustrate the transmission function (TF) and the density of states (DOS) for the ground-state asymmetric (left) and symmetric (right) structures in correlation with the frontier molecular orbitals and their eigenvalues (horizontal lines on the left). From these plots, it is clear that a peak in the TF, that is to say, an open transmission channel, corresponds to one delocalized molecular orbital. When such orbitals are well localized, for instance at one end of the system, they do not contribute to the TF. For example, LUMO and LUMO +1 orbitals, both for the asymmetric and symmetric structures (a), do not contribute to the TF, whereas LUMO +2 is sufficiently delocalized to open a transport channel. Degenerate or sufficiently close eigenvalues may contribute to a single transmission channel.
Furthermore, within the energy window of $\pm 2 \mathrm{eV}$ shown in Fig. 6, the TF for the symmetric structure (a) is considerably larger (note the logarithmic scale!) than for the asymmetric structure (a). This fact is an immediate consequence of the degree of localization of the corresponding orbitals. The energy difference between orbitals also plays an important role: The closer the two orbital energies, the higher will be the mixing of these orbitals as they contribute to an open transmission channel. In the symmetric case, the energy difference between the two lowest depicted occupied orbitals (HOMO -1 and HOMO - 2) is $0.36 \mathrm{eV}$, while for the asymmetric structure this difference is $0.53 \mathrm{eV}$. Therefore, the lower depicted orbital, which is more delocalized in the symmetric case, will contribute in this case strongly to the TF.

Figures 7 and 8 show the transmission functions and the I-V characteristics, respectively, of the asymmetrically connected structures (a), (b), (b'), (c) (left panels) and the symmetrically connected structures (a), (b), (c) (right panels). In a window of energies between -2 and $+2 \mathrm{eV}$, the asymmetric structures (upper left panel of Fig. 7) have very small transmission functions: Only the peak just above $-2 \mathrm{eV}$ for structure $\left(\mathrm{b}^{\prime}\right)$ and the peak just above $+2 \mathrm{eV}$ for structure (c) lead to a weak conductance. Remembering that by Eq. 10, in the zero-temperature limit, the current $I(V)$ is given by integrating the TF from $-V / 2$ to $+V / 2$, we can immediately deduce from the left panels of Fig. 7 that the onset of the current should be just below $4 \mathrm{~V}$ for structure $\left(b^{\prime}\right)$ and just above $4 \mathrm{~V}$ for structure (c). This 
Fig. 5 HOMO and LUMO for the extended structures $(a),(b)$, $\left(b^{\prime}\right)$ and $(c)$. In the asymmetric structures $(b)$ and $(c)$, the HOMOs are localized in a region of the molecule diagonally opposite to the HOMOs of $(a)$ and $\left(b^{\prime}\right)$. Likewise, the LUMOs of the asymmetric structure $(b)$ and $(c)$ are localized diagonally opposite to the LUMOs of $(a)$ and $\left(b^{\prime}\right)$. Since in $b$ and $c$ the hydrogen $\mathrm{H}^{\prime}$ points to the opposite direction compared with $(a)$ and $\left(b^{\prime}\right)$, the redistribution of these orbitals is associated with the rotation of $\mathrm{H}^{\prime}$

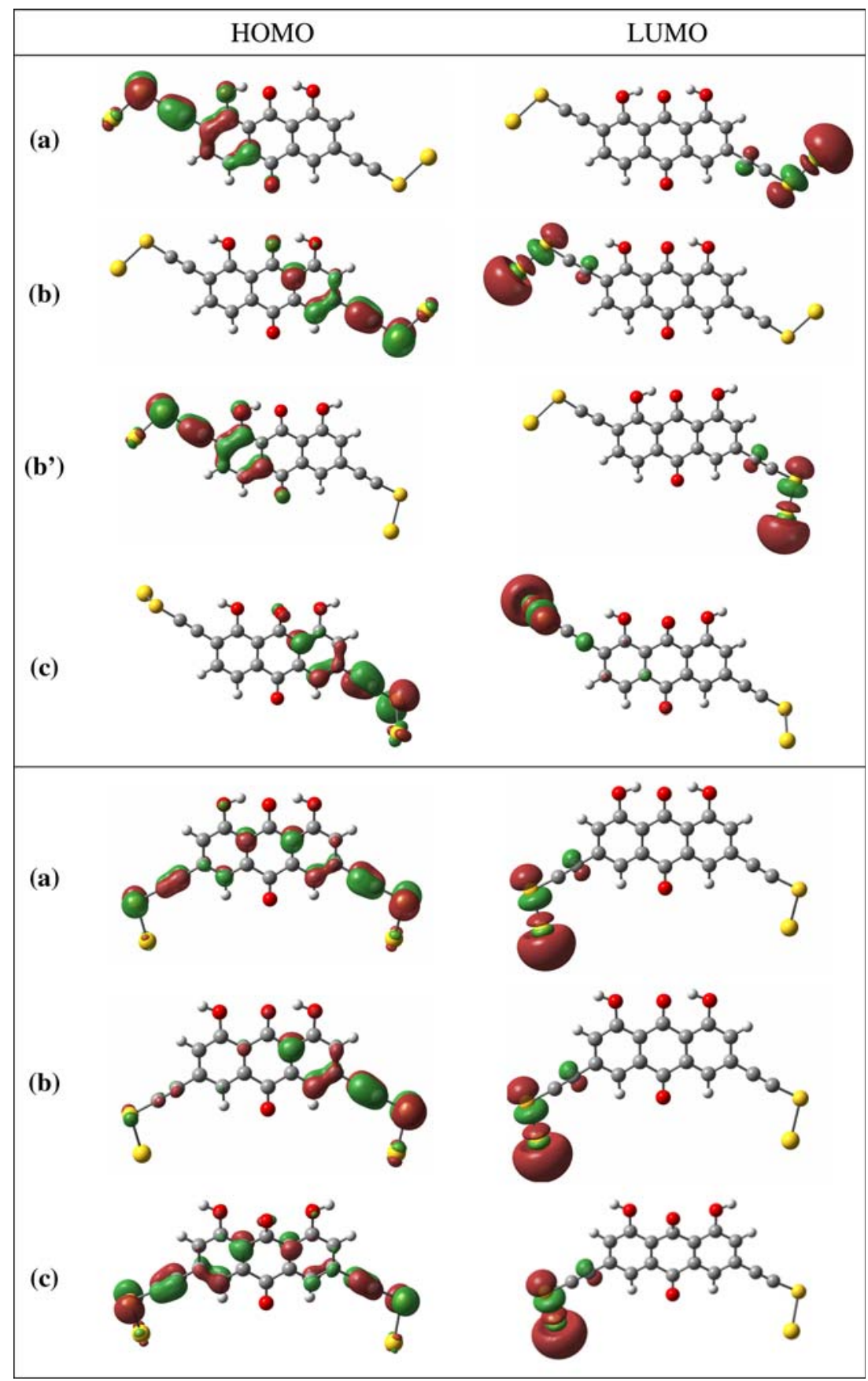

is exactly what Fig. 8 shows. A significant increase of the current through the asymmetrically connected structures is found only for structures (a) and (c), if the voltage is above $6 \mathrm{~V}$. This increase in the current is a consequence of the additional conduction channels visible in the lower left panel of Fig. 7.

For the symmetrically connected structures, the onset of the current (see Fig. 8, right panel) is found at a much lower bias $(2 \mathrm{~V})$ than for the asymmetric structures. This follows immediately from the strong conduction through the occupied orbitals visible just below $-1 \mathrm{eV}$ in the right- hand panels of Fig. 7. This result demonstrates clearly that if the geometry of the molecular junction is not known experimentally, it is possible to assign the conformer present in the junction by its transmission properties. The onset of the current, appearing at $4 \mathrm{~V}$ for the asymmetric structures versus $2 \mathrm{~V}$ for the symmetric ones, clearly shows in which geometry the chrysazine molecule is connected to the leads.

The I-V characteristics of the symmetrically connected conformers show an additional peculiarity, which is highly interesting: within a bias range from 3.5 to $4.2 \mathrm{~V}$, the I-V 

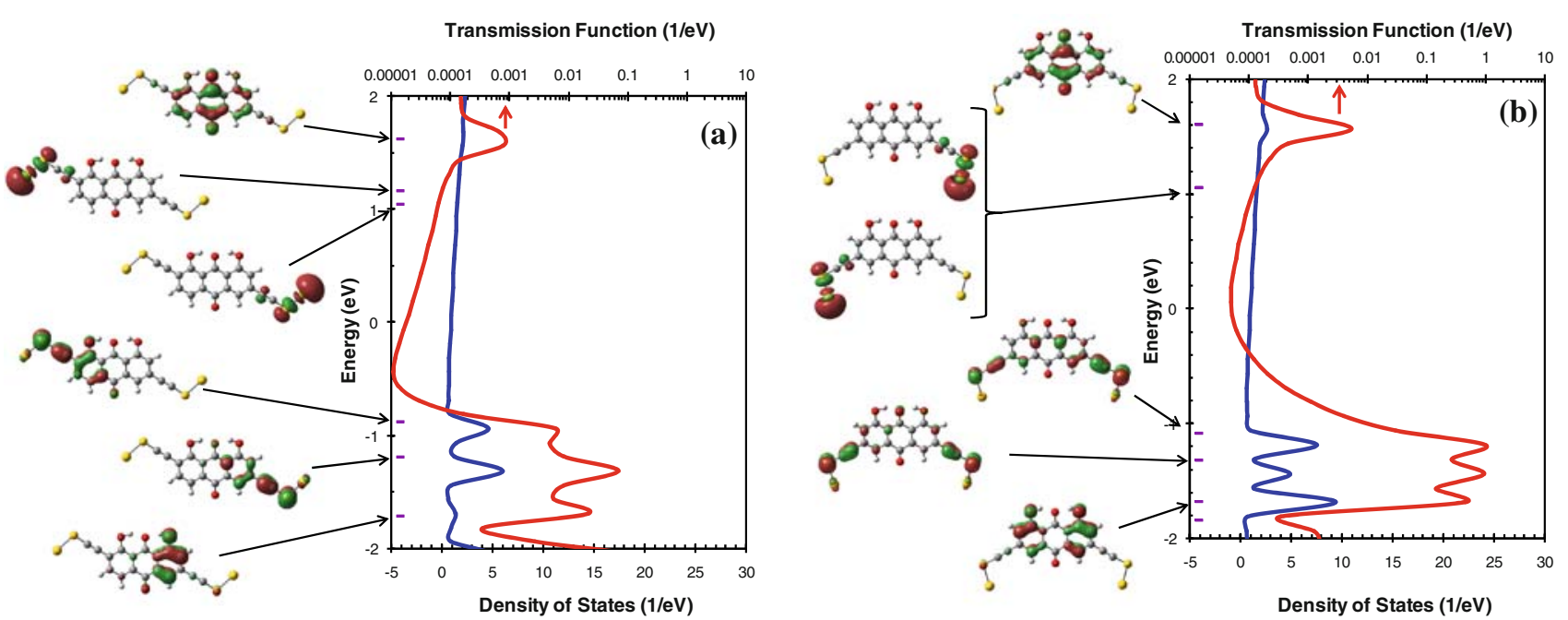

Fig. 6 Transmission function (red) and density of states (blue) together with the corresponding frontier molecular orbitals for the extended structures $(a)$ of Fig. 4. The Kohn-Sham eigenvalues of the extended systems are shown on the left in lilac
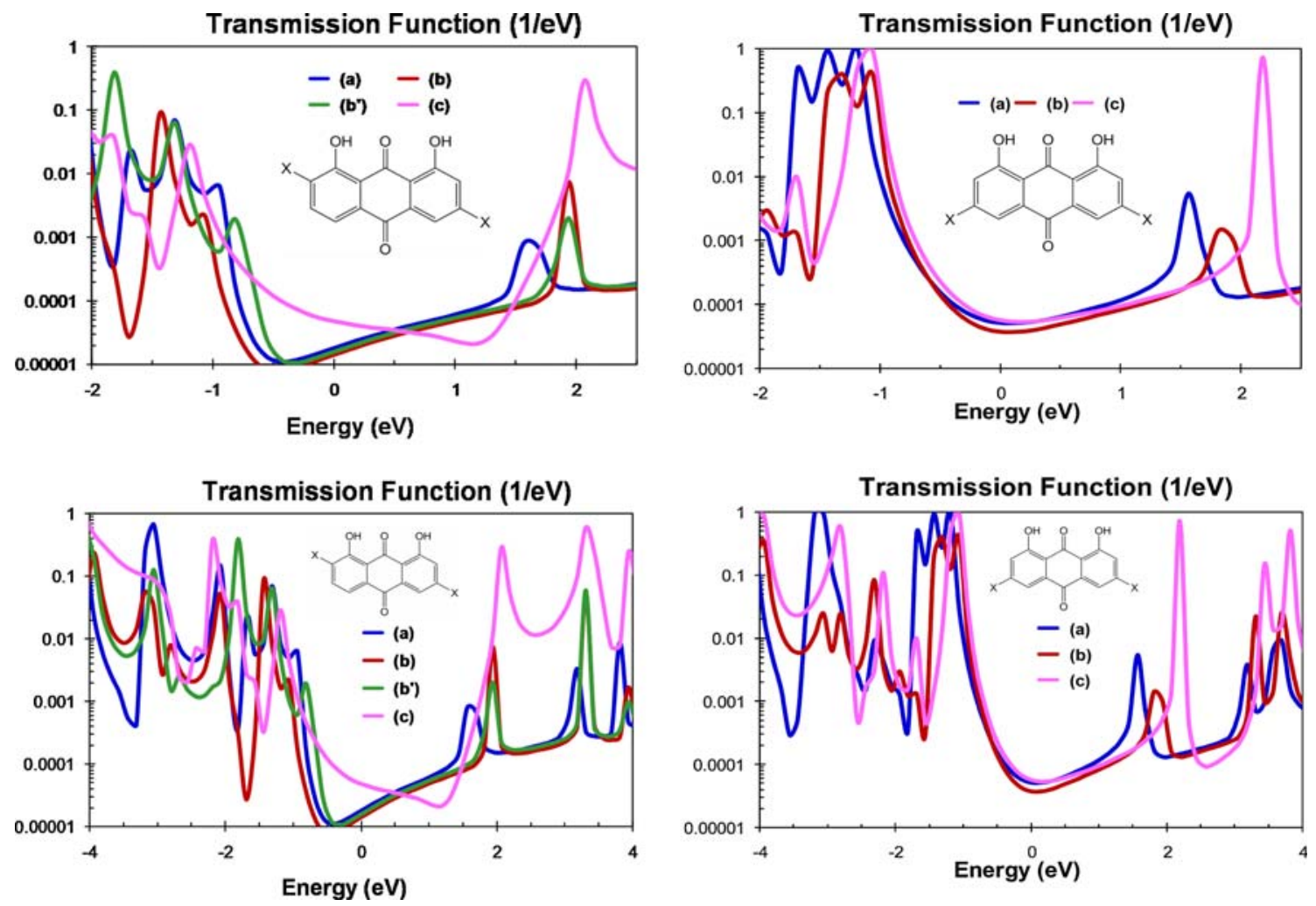

Fig. 7 Transmission functions for the extended structures shown in Fig. $4 ; a$ in blue, $b$ red, $b^{\prime}$ green, and $c$ pink. The upper panels show the transmission functions for the asymmetric (left) and symmetric

curves of the structures (b) and (c) are constant and nearly identical, while structure (a), within the same bias window, shows a constant current nearly twice as large. This suggests the use of chrysazine as a molecular optical switch: if an isomerization from structure (a) to structure (b) or (c) can be achieved by a suitable laser pulse, this will lead to a (right) structures within the energy window $\pm 2 \mathrm{eV}$. The lower panels show the same transmission functions in the larger energy window $\pm 4 \mathrm{eV}$

well-defined jump in the current if the device is operated at a voltage between 3.5 and $4.2 \mathrm{~V}$.

In summary, our results reveal that the various isomers of chrysazine and their possible connections to the leads have significantly different transport properties. This allows one to assign experimental $\mathrm{I}-\mathrm{V}$ characteristics to 

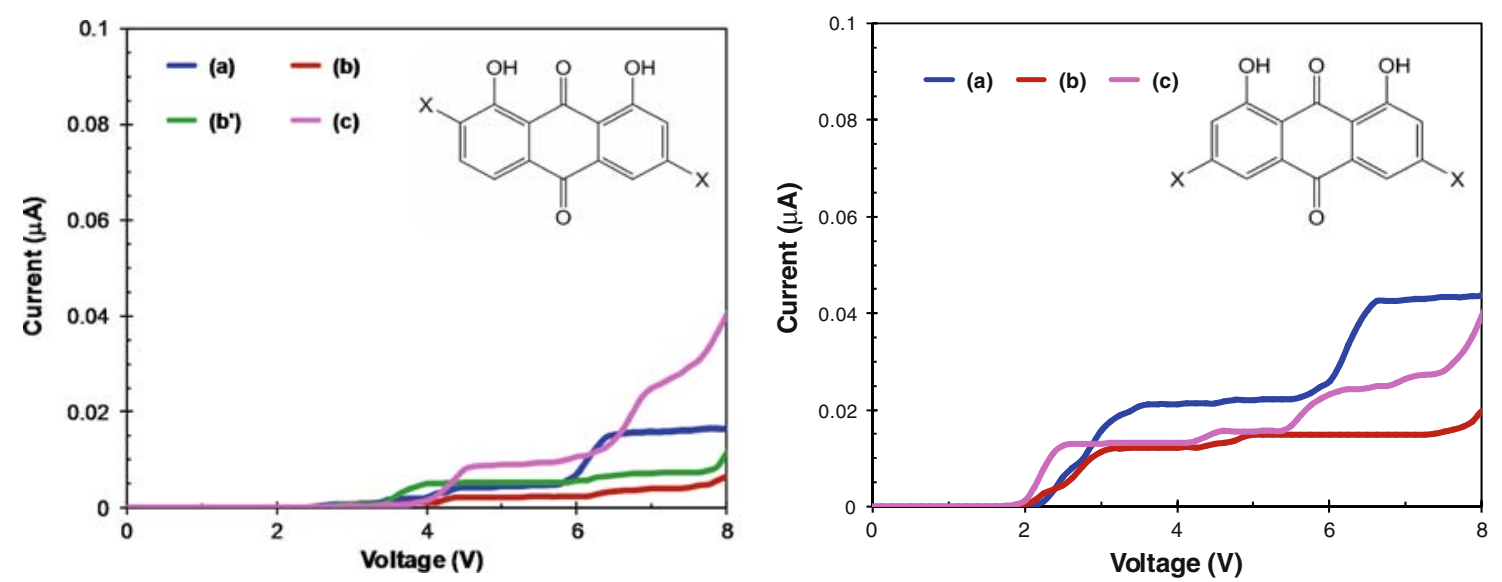

Fig. 8 Current-voltage characteristics for the extended structures $(a),(b),\left(b^{\prime}\right)$ and $(c)$

specific conformers and furthermore suggests that chrysazine may be used as a molecular optical switch.

Acknowledgments This work was supported by the Deutsche Forschungsgemeinschaft within the SFB 658 and by the e-I3 ETSF project (INFRA-2007-1.2.2: Grant Agreement Number 211956). We acknowledge the computational facilities of the HLRN where the calculations were performed.

Open Access This article is distributed under the terms of the Creative Commons Attribution Noncommercial License which permits any noncommercial use, distribution, and reproduction in any medium, provided the original author(s) and source are credited.

\section{References}

1. Datta S (1995) Electronic transport in mesoscopic systems. Cambridge University Press, Cambridge

2. Di Ventra M, Lang ND (2000) Phys Rev Lett 84:979

3. Tian W, Datta S, Hong S, Reifenberger R, Henderson JI, Kubiak CP (1998) J Chem Phys 109:2874

4. Derosa PA, Seminario JM (2001) J Phys Chem B 105:471

5. Hall LE, Reimers JR, Hush NS, Silverbrook K (2000) J Chem Phys 112:1510

6. Chen H, Lu JQ, Wu J, Note R, Mizuseki H, Kawazoe Y (2003) Phys Rev B 67:113408

7. Evers F, Weigend F, Koentop M (2004) Phys Rev B 69:235411

8. Ellenbogen JC, Love JC (2000) Proc IEEE 88:386 (Among others)

9. Mujica V, Kemp M, Ratner MA (1994) J Chem Phys 101:6849

10. Mujica V, Kemp M, Ratner MA (1994) J Chem Phys 101:6856
11. Vondrak T, Cramer CJ, Zhu X-Y (1999) J Phys Chem B 103:8915

12. Seminario JM, Zacarias AG, Derosa PA (2002) J Chem Phys 116:1671

13. Frisch MJ, Trucks GW, Schlegel HB, Scuseria GE, Robb MA, Cheeseman JR, Montgomery Jr JA, Vreven T, Kudin KN, Burant JC, Millam JM, Iyengar SS, Tomasi J, Barone V, Mennucci B, Cossi M, Scalmani G, Rega N, Petersson GA, Nakatsuji H, Hada M, Ehara M, Toyota K, Fukuda R, Hasegawa J, Ishida M, Nakajima T, Honda Y, Kitao O, Nakai H, Klene M, Li X, Knox JE, Hratchian HP, Cross JB, Bakken V, Adamo C, Jaramillo J, Gomperts R, Stratmann RE, Yazyev O, Austin AJ, Cammi R, Pomelli C, Ochterski JW, Ayala PY, Morokuma K, Voth GA, Salvador P, Dannenberg JJ, Zakrzewski VG, Dapprich S, Daniels $\mathrm{AD}$, Strain MC, Farkas O, Malick DK, Rabuck AD, Raghavachari K, Foresman JB, Ortiz JV, Cui Q, Baboul AG, Clifford S, Cioslowski J, Stefanov BB, Liu G, Liashenko A, Piskorz P, Komaromi I, Martin RL, Fox DJ, Keith T, Al-Laham MA, Peng CY, Nanayakkara A, Challacombe M, Gill PMW, Johnson B, Chen W, Wong MW, Gonzalez C, Pople JA (2004) Gaussian 03, Revision D.01, Gaussian Inc, Wallingford, CT

14. Becke AD (1993) J Chem Phys 98:5648

15. Perdew JP, Chevary JA, Vosko SH, Jackson KA, Pederson MR, Singh DJ, Fiolhais C (1992) Phys Rev B 46:6671

16. Perdew JP, Chevary JA, Vosko SH, Jackson KA, Pederson MR, Singh DJ, Fiolhais C (1993) Phys Rev B 48:4978

17. Perdew JP, Burke K, Wang Y (1996) Phys Rev B 54:16533

18. Arzhantsev SY, Takeuchi S, Tahara T (2000) Chem Phys Lett 330:83 and references therein

19. Smulevich G, Marzocchi MP (1985) Chem Phys 94:99

20. Zain SM, Ng SW (2005) Acta Crystallogr E61:02921

21. Kaun C-C, Seideman T (2008) Phys Rev B77:033414

22. Seminario JM, Zacarias AG, Tour JM (1999) J Am Chem Soc $121: 411$ 